\title{
Nanotheranostics
}

2018; 2(4): 295-305. doi: 10.7150/ntno.22544

Research Paper

\section{Near-Infrared Activated Release of Doxorubicin from Plasmon Resonant Liposomes}

\author{
Shellie S. Knights-Mitchell and Marek Romanowski ${ }^{\bowtie}$ \\ Department of Biomedical Engineering, University of Arizona, 1657 E Helen St, Tucson, AZ 85721, USA \\ $\bowtie$ Corresponding author: marekrom@email.arizona.edu 520-626-1578 \\ (C) Ivyspring International Publisher. This is an open access article distributed under the terms of the Creative Commons Attribution (CC BY-NC) license \\ (https://creativecommons.org/licenses/by-nc/4.0/). See http://ivyspring.com/terms for full terms and conditions.
}

Received: 2017.09.18; Accepted: 2018.05.25; Published: 2018.06.21

\begin{abstract}
Precise control of drug release from nanoparticles can improve efficacy and reduce systemic toxicity associated with administration of certain medications. Here, we combined two phenomena, photothermal conversion in plasmon resonant gold coating and thermal sensitivity of liposome compositions, to achieve a drug delivery system that rapidly releases doxorubicin in response to external stimulus.

Methods: Thermosensitive liposomes were loaded with doxorubicin and gold-coated to produce plasmon resonant drug delivery system. Plasmon resonance facilitates release of contents upon near-infrared laser illumination, thus providing spatial and temporal control of the process. This controlled delivery system was compared to thermosensitive liposomes without gold coating and to the FDA-approved Doxil that was gold-coated to create a plasmon resonant coating. Release of doxorubicin from the gold-coated thermosensitive liposomes was further confirmed by tests of cell viability.

Results: Upon laser illumination at $760 \mathrm{~nm}$ and $88 \mathrm{~mW} / \mathrm{cm}^{2}$ power density, permeability of plasmon resonant liposomes increased by three orders of magnitude, from $70 \times 10^{-12}$ to $60,000 \times 10^{-12} \mathrm{~cm} / \mathrm{s}$. In control experiments, mild hyperthermia $\left(42^{\circ} \mathrm{C}\right)$ increased permeability of these thermosensitive liposomes to just $3,700 \times 10^{-12} \mathrm{~cm} / \mathrm{s}$. Neither hyperthermia nor laser illumination elicit content release from Doxil or plasmon resonant Doxil obtained by gold coating. Laser-induced release of doxorubicin from plasmon resonant thermosensitive liposomes resulted in the loss of cell viability significantly greater than in any of the control groups.

Conclusion: Combination of thermosensitive liposomes with plasmon resonant coating enables rapid, controlled release, not currently available in pharmaceutical formulations of anticancer drugs.
\end{abstract}

Key words: liposomes, doxorubicin, controlled release, drug delivery, plasmon resonance

\section{Introduction}

With nearly 2 million people diagnosed with cancer in the US in 2017, the overall improvement of efficiency of treatment, while notable, is below the NCI strategic goals of eliminating death and suffering due to cancer by 2015 [1,2]. Among the key treatment modalities, chemotherapy is particularly disappointing, contributing just about $2 \%$ to 5 year survival in all types of cancers [3]. While producing lasting remission in certain cancers such as acute lymphocytic leukemia and gestational choriocarcinoma, the use of many chemotherapeutic agents is limited by their very narrow therapeutic window, i.e., the range of doses that are simultaneously efficacious and non-toxic [4,5]. New formulations of established chemotherapeutic agents, including Doxil, a liposomal formulation of doxorubicin, have been introduced to clinical practice in the US to limit systemic exposure to such drugs $[6,7]$. Reduction of cardiotoxicity associated with doxorubicin is an added advantage of drug 
encapsulation within the liposome [8,9]. Moreover, this delivery system allows for successful drug accumulation at the tumor site via the enhanced permeability and retention (EPR) effect that is made possible by the leaky vasculature and decreased lymphatic drainage characteristic of the tumor environment $[8,10]$.

Further improvement of efficacy can be achieved by targeted delivery and controlled release. Targeted delivery mechanisms rely on molecular recognition of various markers overexpressed in diseased cells $[11,12]$. In controlled release, the delivered therapeutic substance is released or activated on demand. Methods of activation may include internal conditions associated with the disease such as a local change of $\mathrm{pH}$, or external stimuli, such as hyperthermia or illumination with near infrared (NIR) light [13-16].

Previously, we introduced and characterized plasmon resonant liposomes, a composite material that upon near infrared illumination releases content with exquisite spatial and temporal control $[17,18]$. In the preparative process, gold nanoparticles were formed by in situ reduction of ionic gold in the presence of phosphatidylcholine liposomes, yielding gold-coated liposomes $[19,20]$. We characterized these preparations by several electron microscopy techniques, confirming formation of gold coating on the surface of liposomes [19], formation of small, 1-2 $\mathrm{nm}$ gold clusters [20], and stability of the resulting structures upon illumination with NIR light [21]. The use of NIR light promotes a greater depth of penetration with reduced damage to tissue [22]. We also introduced a computational model to relate the resulting plasmon resonance spectra to the density and size of gold cluster on the liposomal template [20]. The preparative process was confirmed independently by others who have investigated plasmon resonant liposomes in vitro and in vivo [23]. We tested this novel technology in several assays requiring delivery of simple molecular probes or ligands [24,25]. Encouraged by these results we hypothesize that the plasmon resonant liposomes can support controlled release of antineoplastic agents used in the treatment of cancer.

In this report we demonstrate on-demand release by activation using NIR light. We accomplished this by encapsulating doxorubicin in thermosensitive plasmon resonant liposomes. Quantitative analysis of content release demonstrates a rapid, switch-like, release in response to the near-infrared trigger stimuli, not achievable in un-coated thermosensitive liposomes or in Doxil, the FDA-approved liposomal formulation of doxorubicin. Precise, on-demand delivery of high doses of drug, while minimizing systemic exposure, would effectively decrease side effects associated with chemotherapy, may increase patient compliance and improve the quality of life of patients [26].

\section{Materials and Methods}

\section{Liposome preparation and characterization}

Liposomes composed of 1,2-dipalmitoyl-snglycero-3-phosphocholine (DPPC), hydrogenated soy L-a-phosphatidylcholine (HSPC), cholesterol, and 1,2-dipalmitoyl-sn-glycero-3-phosphoethanlamine-N[methoxy(polyethyleneglycol)-2000] (ammonium salt) (DPPE-PEG 2000) (Avanti Polar Lipids, Alabaster, $\mathrm{AL}$ ), were prepared in the molar ratio 50:25:15:3 using a method similar to that described by de Smet et al. (Figure 1) [27]. This combination of lipids was specifically chosen because it resulted in a thermosensitive formulation. Lipids and cholesterol were weighed, mixed and completely dissolved in chloroform. Most of the chloroform was removed by exposure to a gentle flow of nitrogen. The sample was left under vacuum overnight for complete removal of the remaining solvent. Lipids were rehydrated using $240 \mathrm{mM}$ ammonium sulfate solution to prepare a 20 $\mathrm{mM}$ lipid suspension. After ten cycles of freeze and thaw using an isopropanol/dry ice bath and $60{ }^{\circ} \mathrm{C}$ water bath, the samples were extruded through polycarbonate membranes (Whatman, Florham Park, NJ), pore size $200 \mathrm{~nm}$ and $100 \mathrm{~nm}, 5$ times each at 60 ${ }^{\circ} \mathrm{C}$ using a $10 \mathrm{ml}$ LIPEX extruder (Northern Lipids Inc., Vancouver, BC). Liposome size was measured using the Nano-ZS Zetasizer from Malvern Instruments.

\section{Drug Loading}

Drug loading was accomplished using previously described remote loading methods $[7,27]$. Un-encapsulated ammonium sulfate was replaced with HEPES buffered saline (HBS) (20 mM HEPES, $137 \mathrm{mM} \mathrm{NaCl}$ ) via gel filtration. Doxorubicin- $\mathrm{HCl}$ (Sigma Aldrich, St. Louis, MO) solution at $5 \mathrm{mg} / \mathrm{ml}$ was added to the liposome suspension at 20:1 phospholipid/drug ratio. The sample was incubated for 90 minutes at $37{ }^{\circ} \mathrm{C}$ after which un-encapsulated drug was removed via gel filtration using a PD-10 desalting column (GE Healthcare Life Sciences, Pittsburg, PA). To determine encapsulation efficiency $(E E)$, unpurified and purified samples were lysed using Triton X-100 and the fluorescence intensity of doxorubicin was collected. The fluorescence intensity of doxorubicin before purification, $I_{\text {total, }}$ and doxorubicin fluorescence intensity after purification, Iliposome, were measured and $E E$ was calculated as:

$$
E E(\%)=I_{\text {liposome }} / I_{\text {total }} \times 100 \%
$$




\section{Gold coating}

Reduction of gold was carried out following a previously reported technique [17]. Briefly, an aqueous solution of gold chloride $(100 \mathrm{mM})$ was added to a sample of liposome suspension $(1 \mathrm{ml}, 10$ $\mathrm{mM}$ lipids), followed by an aqueous solution of ascorbic acid $(500 \mathrm{mM})$. Each addition was accompanied by gentle swirling until a distinct color change, attributed to plasmon resonance, was observed. For resonant peak wavelengths at $760 \mathrm{~nm}$, $24 \mu \mathrm{l}$ gold chloride solution was added to the suspension, followed by $36 \mu \mathrm{l}$ ascorbic acid solution. Absorbance spectra were obtained using the Cary 5 dual beam spectrophotometer.

\section{Thermal Release}

Thermal release was demonstrated using a water bath, first at $37{ }^{\circ} \mathrm{C}$ and then at $42{ }^{\circ} \mathrm{C}$, to mimic body temperature and moderate hyperthermia respectively. Liposomes were diluted to $150 \mu \mathrm{M}$ lipids using HBS, and $2 \mathrm{ml}$ of the resulting suspension was transferred to a cuvette. The cuvette was placed in the preheated water bath for a predetermined length of time. Subsequently, fluorescence emission spectra of the samples were collected using the QE65000 Spectrometer (Ocean Optics, Dunedin, FL) with a 470 $\mathrm{nm}$ LED providing excitation light. Triton X-100 was added to lyse liposomes and obtain fluorescence emission for $100 \%$ content release. The percent of drug release was calculated as:

$$
\text { drug release }(\%)=\left(I-I_{0}\right) /\left(I_{\tau}-I_{0}\right) \times 100 \%
$$

Where $I$ is the sample maximum intensity of fluorescence emission at $597 \mathrm{~nm}$ (excitation at 470 $\mathrm{nm}), I_{0}$ is the maximum emission of an untreated sample, and $I_{\tau}$ is the maximum emission after treatment with Triton X-100.

\section{Light-Induced Release}

Light-induced release was tested in all the following liposome formulations: uncoated doxorubicin-loaded liposomes (hereafter LU), gold-coated doxorubicin-loaded liposomes (hereafter LC), Doxil (Doxorubicin Hydrochloride Liposome Injection, Northstar Rx LLC, Memphis, TN, hereafter DU) and gold-coated Doxil (hereafter DC). All samples were illuminated with a $760 \mathrm{~nm}$ laser diode (RPMC Lasers, O'Fallon, MO) driven by a constant current source (ILX Lightwave, Bozeman, MT) using a method previously described $[17,20]$. A $30 \mu l$ as prepared sample was placed in a cuvette. The sample was illuminated for the desired amount of time (from 0 to 5 minutes) with the diode laser operating at $0.5 \mu \mathrm{s}$ pulse width and $10 \%$ duty cycle. Liposomes were diluted to $2 \mathrm{ml}(150 \mu \mathrm{M})$ and fluorescence emission spectrum was collected. This was followed by the lysis of liposomes by Triton X-100 and a second measurement of fluorescence emission. The percent of drug released was calculated using Equation 2.

\section{Permeability Coefficients}

Assuming that doxorubicin release is the result of diffusion only, the permeability coefficient, $P$, is calculated using Equation 3 where $r$ is the internal radius of the liposome and $k$ is the first order rate constant [28]:

$$
P=(r / 3) \times k
$$

To determine $k$, the release data was fitted to a first order release equation (Equation 4) where $F$ represents the percent drug released, $M_{o}$ represents the total amount of drug released, $k$ represents the first order rate constant and $t$ represents time.

$$
F(\%)=M_{\mathrm{o}} \times\left(1-\mathrm{e}^{-k t}\right)
$$

$M_{o}$ was treated as a variable because the maximum percent of doxorubicin released across experiments varied. For samples that had less than 10 $\%$ release after exposure to stimuli, $M_{o}$ was taken as $100 \%$. For samples that reached a steady maximum, $M_{o}$ was taken as that maximum percent release value.

\section{Differential Scanning Calorimetry (DSC)}

DSC was performed using the NanoDSC from TA Instruments (New Castle, DE). Each sample was dialyzed against HEPES buffer before experimentation. This equipment utilizes a capillary cell design to decrease the potential for sample aggregation and solid-state thermoelectric elements that allow for a high degree of sample temperature control. Thermal analysis was conducted at a 1 ${ }^{\circ} \mathrm{C} /$ min heating rate from $10-90{ }^{\circ} \mathrm{C}$ with a $600 \mu \mathrm{l}$ sample volume. Analysis was completed by normalizing data to the mass of phospholipid present in each sample and correcting for baseline drift by a sigmoidal baseline subtraction. The TA instruments software, NanoAnalyze was used for data analysis. The data were fit to Gaussian distributions to obtain thermodynamic parameters including transition temperature $\left(T_{m}\right)$, temperature width of the transition at half the peak height $\left(\Delta T_{1 / 2}\right)$ and calorimetric enthalpy $\left(\Delta H_{\text {cal }}\right)$. The values of $T_{m}$ and $T_{1 / 2}$ and the molar gas constant $(R)$ were used in Equation 5 to determine an approximate value of the van't Hoff enthalpy $\left(\Delta H_{V H}\right)$ :

$$
\Delta H_{V H} \approx 4 R T_{m^{2}} / \Delta T_{1 / 2}
$$

Consequently, the value of the cooperative unit $(\mathrm{CU})$ for each lipid composition was calculated as $\mathrm{CU}$ $=\Delta H_{V H} / \Delta H_{\text {cal }}$. 


\section{Cell Viability and Fluorescence Imaging}

A375 (human melanoma) cells were seeded on a 96 well plate at a seeding density of $1 \times 10^{5}$ cells $/ \mathrm{ml}$ in RPMI $(10 \%$ fetal bovine serum $(v / v), 1 \%$ penicillin-streptomycin $(v / v))$ and were left to attach overnight at $37{ }^{\circ} \mathrm{C}$ and $5 \% \mathrm{CO}_{2}$. Liposome formulations and free doxorubicin were exposed to different stimuli before being added to cells hyperthermia at $42{ }^{\circ} \mathrm{C}$ for 20 minutes or laser illumination at $760 \mathrm{~nm}$ for 2 minutes. Cells were then treated with four different sets of doxorubicin liposome formulations. Samples of formulations were diluted in fully supplemented RPMI to a final doxorubicin concentration of $10 \mu \mathrm{M} .100 \mu \mathrm{l}$ of doxorubicin loaded liposomes diluted in cell culture media was added to each well and cells were left to incubate for 4 hours in a humidified chamber at $37{ }^{\circ} \mathrm{C}$ and $5 \% \mathrm{CO}_{2}$. Controls included cells that were untreated (control), cells that were exposed to the drug but without any stimulus (no stimulus) and free doxorubicin (free). After incubation, $20 \mu \mathrm{l}$ resazurin dye $(0.15 \mathrm{mg} / \mathrm{ml})$ was added to each well and the cells were incubated in a humidified chamber at $37^{\circ} \mathrm{C}$ for 2 hours. The resulting resorufin signal (pink and fluorescent) obtained from metabolically active cells was measured using a fluorimeter with excitation wavelength of $531 \mathrm{~nm}$ and emission wavelength of $615 \mathrm{~nm}$. The average and standard deviation from eight wells were calculated and cell viability was determined as a percentage that was based on the fraction of cells alive in the treated samples compared to untreated cells (control).

A<smiles>CCCCCCCCCCCCCCCC(=O)OCCCOP(=O)(O)OCC[N+](C)(C)C</smiles>

B

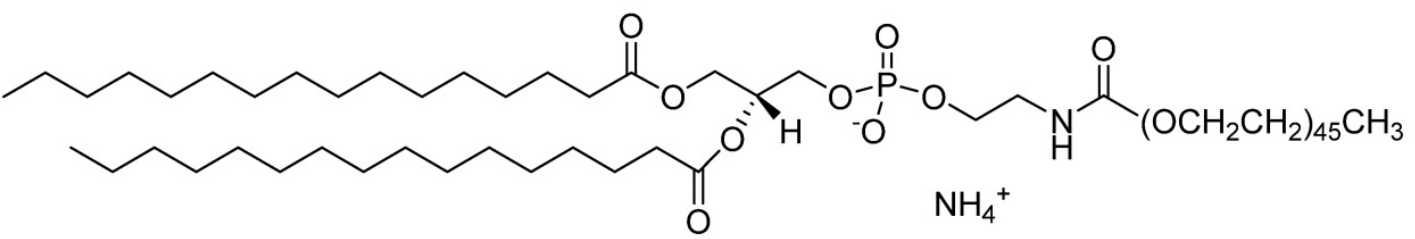

C<smiles>CC(C)CCC[C@H](C)[C@H]1CC[C@H]2[C@@H]3CC=C4C[C@H](O)CC[C@]4(C)[C@H]3CC[C@@]21C</smiles>

D<smiles>COc1cccc2c1C(=O)c1c(O)c3c(c(O)c1C2=O)C[C@@](O)(C(=O)CO)C[C@H]3O[C@H]1C[C@H](N)[C@@H](O)[C@H](C)O1</smiles>

E

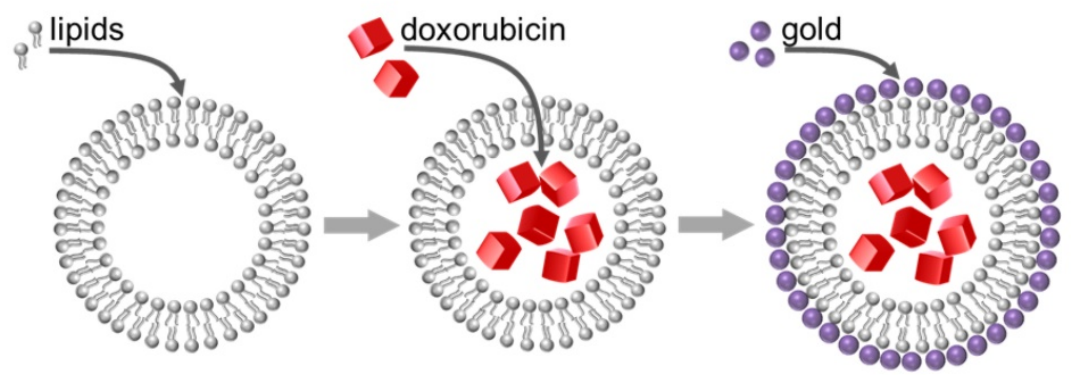

Figure 1. Molecular structures of lipids,1,2-dipalmitoyl-sn-glycero-3-phosphocholine (DPPC) (A) and 1,2-dipalmitoyl-sn-glycero-3-phosphoethanlamine-N-[methoxy (polyethyleneglycol)-2000] (DPPE PEG 2000) (B) and cholesterol (C) used in the formation of liposomes. (D) Chemical structure of doxorubicin-HCl - drug encapsulated in liposomes. (E) Unilamellar liposomes are prepared by freeze-thaw followed by extrusion, loaded with doxorubicin by the ammonium gradient method, and coated with gold by in-situ reduction of gold chloride. 
Microscopy images were collected using an inverted microscope (IX71, Olympus, Center Valley, Pennsylvania) equipped with a 60X 1.42 NA oil-immersion lens (Olympus, Center Valley, PA) and an air-cooled 512×512 pixel EM-CCCD digital camera (Hamamatsu, Bridgewater, NJ). Cells were visualized by differential interference contrast, then by epifluorescence images of cell nuclei followed by doxorubicin. The cell nuclei were visualized by staining with Hoechst 33328 dye and using a filter cube comprising a 350/25 nm excitation bandpass filter, $400 \mathrm{~nm}$ long pass dichroic mirror, and a $420 \mathrm{~nm}$ long pass filter for emission collection. Doxorubicin fluorescence was collected using a 480/20 nm excitation bandpass filter, $565 \mathrm{~nm}$ long pass dichroic mirror and a 605/35 nm emission bandpass filter.

\section{Statistical Analysis}

GraphPad Prism version 7.03 for Windows (GraphPad Software, La Jolla, CA) was used for all statistical analysis. Differences in effect of the multiple liposomal formulations on cell viability were analyzed using one-way ANOVA followed by Dunnett's multiple comparisons test. A $p$ value $<0.05$ was considered significant.

\section{Results}

\section{Doxorubicin-loaded liposome characterization}

Liposomes were loaded with doxorubicin using an ammonium sulfate gradient for an active loading process. These liposomes (LU and LC) were compared to Doxil (DU and DC). Both sets of formulations encapsulated the anthracycline drug doxorubicin, however, a thermosensitive lipid composition was selected for LU and LC (Table 1).

As shown in Table 2, the hydrodynamic diameter of LU determined by dynamic light scattering was $123 \mathrm{~nm}$. On the addition of gold to the

A

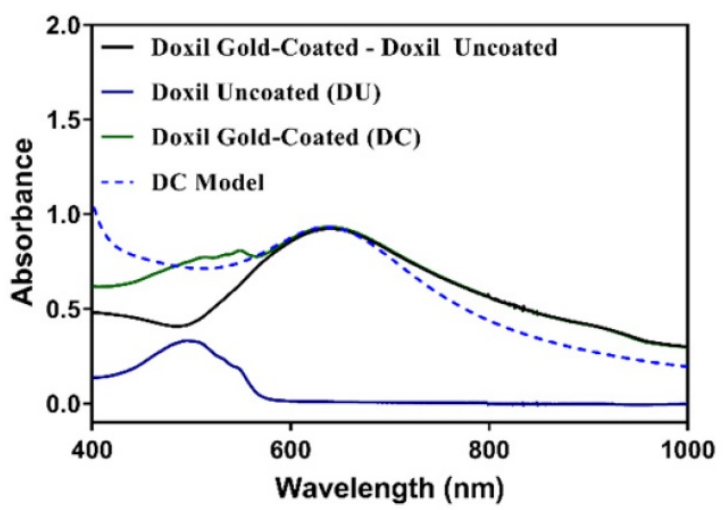

liposome surface to generate LC, the apparent diameter increased to $158 \mathrm{~nm}$. Uncoated Doxil (DU) had a slightly smaller diameter at $86 \mathrm{~nm}$ while its gold coated counterpart followed a similar trend where the plasmon resonant Doxil (DC) diameter increased to $142 \mathrm{~nm}$ (Table 2).

Table 1. Lipid composition (mol \%) of prepared liposomes (LU and LC) vs. Doxil (DU and DC). 1,2-dipalmitoyl-sn-glycero-3phosphocholine (DPPC), hydrogenated soy L-a-phosphatidylcholine (HSPC), cholesterol (CHOL), and 1,2-dipalmitoyl-snglycero-3-phosphoethanlamine-N-[methoxy(polyethyleneglycol)2000] (DPPE-PEG 2000), and 1,2-distearoyl-sn-glycero-3phosphoethanlamine- $\mathrm{N}$-[methoxy(polyethyleneglycol)-2000] (DSPE-PEG 2000).

\begin{tabular}{lll}
\hline Lipid & Liposomes $(\mathrm{mol} \%)$ & Doxil $(\mathrm{mol} \%)$ \\
\hline DPPC & 50 & 0 \\
HSPC & 25 & 55 \\
CHOL & 15 & 40 \\
DPPE-PEG2000 & 3 & 0 \\
DSPE-PEG2000 & 0 & 5 \\
\hline
\end{tabular}

Table 2. Liposome characterization summary. Hydrodynamic diameter measured via dynamic light scattering.

\begin{tabular}{lll}
\hline Sample Name & Hydrodynamic Diameter $(\mathrm{nm})^{*}$ & PDI $^{*}$ \\
\hline Doxil Uncoated (DU) & $86 \pm 4$ & $0.110 \pm 0.130$ \\
Doxil Gold-Coated (DC) & $142 \pm 42$ & $0.600 \pm 0.270$ \\
Liposomes Uncoated (LU) & $123 \pm 6$ & $0.084 \pm 0.074$ \\
Liposomes Gold-Coated (LC) & $158 \pm 26$ & $0.390 \pm 0.280$ \\
\hline${ }^{*}$ Mean \pm standard deviation (n=3), PDI = Polydispersity index &
\end{tabular}

This change can be partially attributed to an increase in the size distribution. The uncoated particles have smaller polydispersity indices (PDIs), indicating narrower size distribution. When compared to their gold-coated counterparts, the PDIs increase substantially with the largest being observed in gold-coated Doxil (DC). The characteristic doxorubicin absorbance peak is seen at $465 \mathrm{~nm}$ while the peaks due to plasmon resonance fall within the NIR region for both samples, with that of DC at 660

B

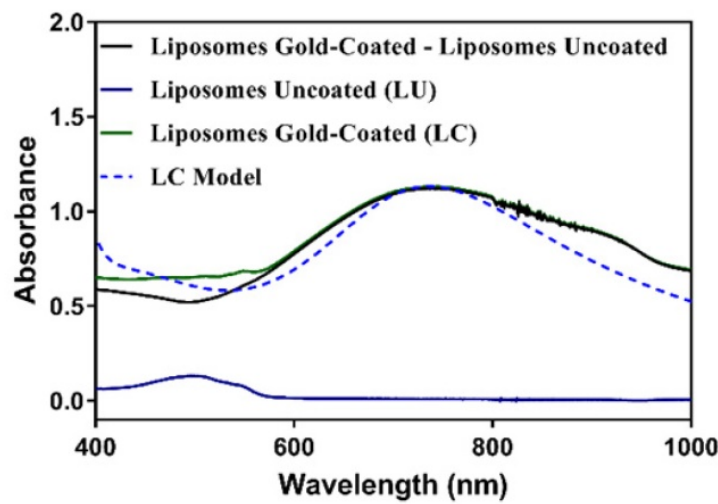

Figure 2. Absorbance spectra of Doxil (A) and thermosensitive liposomes (B): doxorubicin-loaded uncoated (solid blue), doxorubicin-loaded and gold-coated (solid green), plasmon resonance spectrum only (solid black), computational model of plasmon resonance spectrum (dotted blue). 
$\mathrm{nm}$ and LC at $760 \mathrm{~nm}$ (Figure 2). Obtained experimental spectra can be interpreted by computational modelling of the plasmon resonance in gold-coated liposomes [19]. The computational model traces in Figure 2 represent plasmon resonance obtained by decorating the outer surface of these liposomes with $2 \mathrm{~nm}$ gold clusters to cover $62 \%$ (for Doxil) or $80 \%$ (for thermosensitive liposomes) of the surface with gold (see Figure 1E for graphical representation). We attribute the lower gold coverage of Doxil to the lower content of phosphatidylcholines, which likely provide nucleation sites for in situ reduction of ionic gold [20]. The active loading of doxorubicin using the ammonium sulfate gradient led to an encapsulation efficiency of $73 \pm 11 \%$ over five independent experiments.

\section{Drug Release}

Stable drug encapsulation at physiological conditions is paramount for the success of a drug delivery system. Encapsulation stability tests at $37{ }^{\circ} \mathrm{C}$ and $\mathrm{pH} 7.4$, intended to mimic body temperature and $\mathrm{pH}$, were conducted. All samples showed stable doxorubicin retention at physiological temperature and $\mathrm{pH}$ with less than $10 \%$ release at $37^{\circ} \mathrm{C}$ (Figure 3 ).
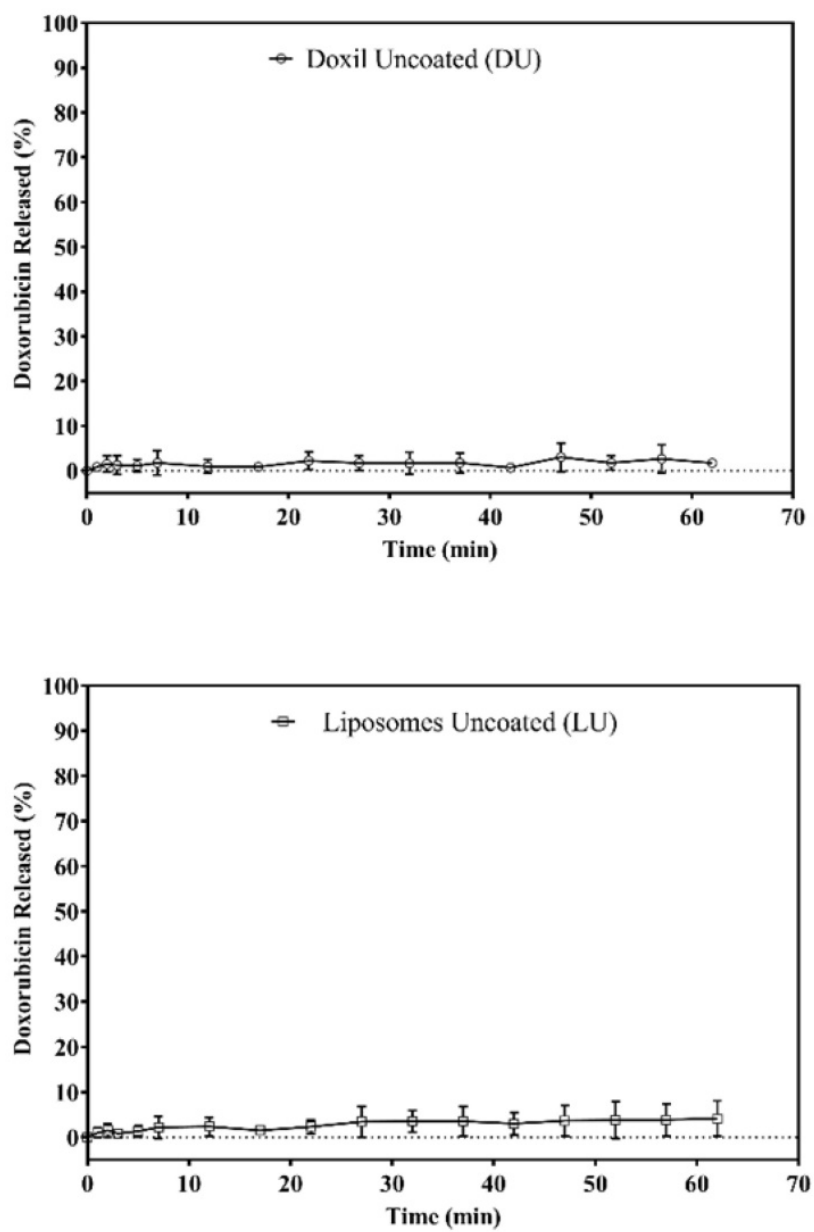

The proposed mechanism requires photothermal conversion in the plasmon resonant gold coating, followed by thermally activated leakage in the thermo-responsive liposomes. Figure 4 shows that both DU and DC were unaffected by the temperature elevation to $42{ }^{\circ} \mathrm{C}$ while LU and LC leaked all their contents within 60 minutes of exposure to hyperthermia.

Finally, to show that plasmon resonant liposomes were responsive to laser illumination, all samples were irradiated at $88 \mathrm{~mW} / \mathrm{cm}^{2}$ power density with a $760 \mathrm{~nm}$ laser diode. The fastest response was obtained from the LC sample after laser exposure, with most of the encapsulated drug being released within 5 minutes of illumination. LC reached the maximum of $74 \%$ release within 5 minutes of laser exposure while there was less than $5 \%$ release from LU over the same period (Figure 5). While both LC and LU displayed significant content release upon hyperthermia at $42{ }^{\circ} \mathrm{C}$, this release was much slower then release from LC initiated by laser illumination. In comparison, release from Doxil (DU and DC) in response to laser illumination and hyperthermia were negligible.
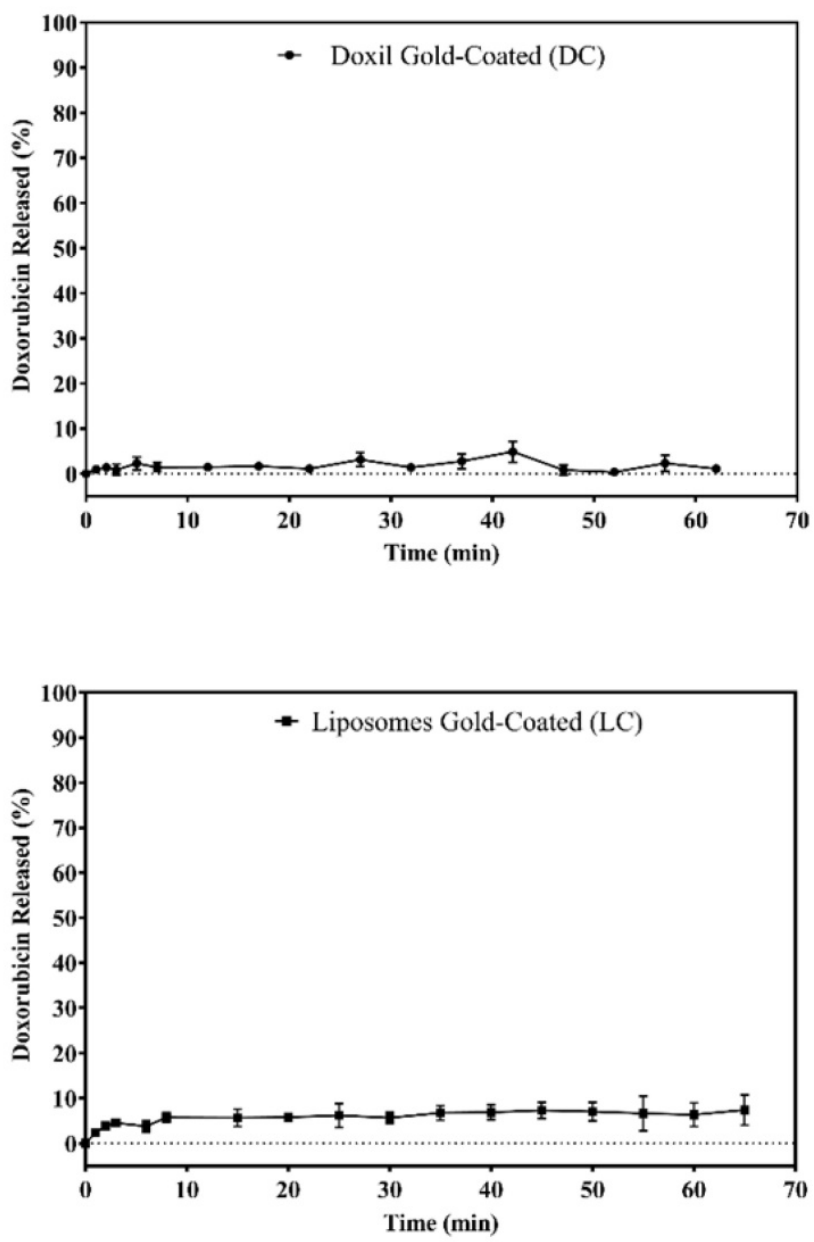

Figure 3. Doxorubicin release at $37 \circ \mathrm{C}$. Doxorubicin release was monitored by measurement of fluorescence intensity. Data are presented as mean \pm standard deviation $(\mathrm{n}=4)$. 


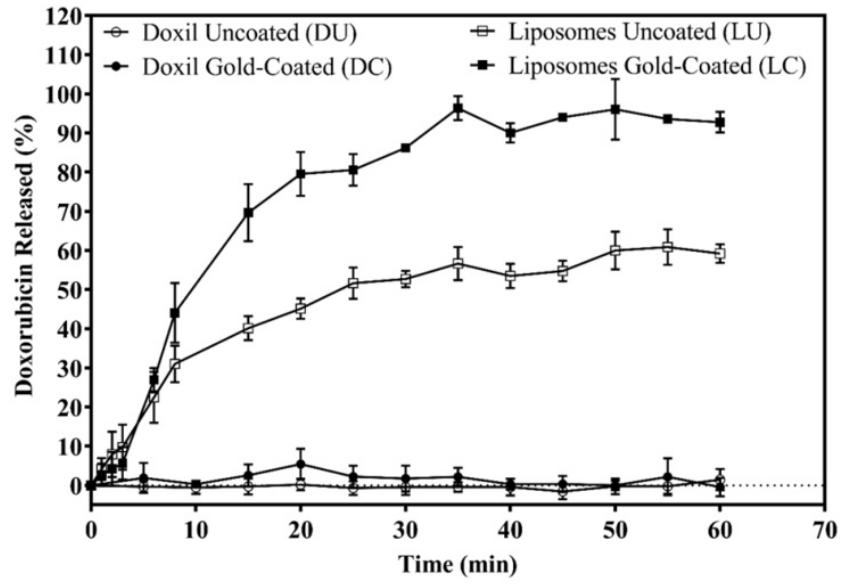

Figure 4. Doxorubicin release at $42{ }^{\circ} \mathrm{C}$. Some of the Doxil Uncoated (DU) and Doxil Gold-Coated (DC) overlap hence in some areas only the DC symbol is seen. Data are presented as mean \pm standard deviation $(n=4)$.

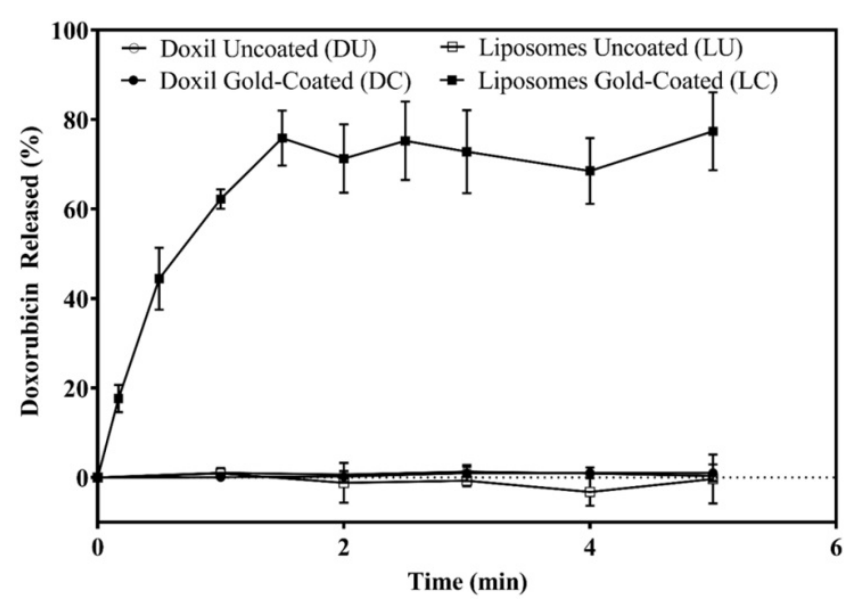

Figure 5. Doxorubicin release via laser illumination. All formulations were exposed to laser illumination at $760 \mathrm{~nm}$. Doxorubicin release was monitored by measurement of fluorescence intensity. Doxil uncoated (DU) and Doxil gold-coated (DC) overlap hence mostly DC points (closed circle) are seen on the graph. Data are presented as mean \pm standard deviation $(n=4)$.

\section{Permeability coefficients}

Permeability coefficients were calculated by fitting the data to a first order release model (Equation 4). The permeability coefficients of the DU and DC liposomes ranged from 10 to $90 \times 10^{-12} \mathrm{~cm} / \mathrm{s}$ at all of the experimental conditions tested, showing negligible response to hyperthermia or laser illumination (Table 3). The LU and LC liposomes displayed much greater responses to stimuli tested. On exposure to mild hyperthermia, the permeability coefficients of LU and LC samples increased similarly, by two orders of magnitude, reaching $2600 \times 10^{-12}$ $\mathrm{cm} / \mathrm{s}$ and $3700 \times 10^{-12} \mathrm{~cm} / \mathrm{s}$ respectively.

Notably, at $42{ }^{\circ} \mathrm{C}$ LC had a slightly higher permeability than LU. The effect of this difference is seen in the release profiles that show a maximum of $60 \%$ release in LU while LC samples had a $90 \%$ maximum release. Exposure to near infrared laser light has a very different impact on the LU and LC samples. It has only mild effect on the permeability of the LU sample, which increased 3-fold compared to its permeability at $37^{\circ} \mathrm{C}$. However, laser illumination of the LC sample resulted in the largest permeability coefficient across all samples, $60,000 \times 10^{-12} \mathrm{~cm} / \mathrm{s}$, a value that is 3 orders of magnitude greater than LC permeability at $37^{\circ} \mathrm{C}$ (Table 3 ).

Table 3. Permeability coefficients of liposome formulations at 37 ${ }^{\circ} \mathrm{C}, 42{ }^{\circ} \mathrm{C}$, and $37{ }^{\circ} \mathrm{C}$ with exposure to $760 \mathrm{~nm}$ laser illumination. Calculations made based on rate constants generated from first order rate equation curve fit to release data.

\begin{tabular}{llll}
\hline & \multicolumn{3}{l}{ Permeability Coefficient, $\mathrm{P}\left(10^{-12} \mathrm{~cm} / \mathrm{s}\right)$} \\
\cline { 2 - 4 } $37{ }^{\circ} \mathrm{C}$ & $42{ }^{\circ} \mathrm{C}$ & Laser \\
\hline Doxil Uncoated (DU) & 10 & 0 & 50 \\
Doxil Gold-Coated (DC) & 20 & 10 & 90 \\
Liposomes Uncoated (LU) & 30 & 2600 & 80 \\
Liposomes Gold-Coated (LC) & 70 & 3700 & 60000 \\
\hline
\end{tabular}

\section{DSC}

In isobaric thermogram scans, the Doxil samples, although non-thermosensitive in our release experiments, exhibited two transitions. One broad, flat transition at $51.6^{\circ} \mathrm{C}$ and $52.3^{\circ} \mathrm{C}$ for the DU and DC samples respectively represented the main lipid phase transition while a sharper, narrower transition at 70 ${ }^{\circ} \mathrm{C}$ was associated with the entrapped doxorubicin crystals (Figure 6). The lipid phase transition in both DU and DC samples had similar $\Delta T_{1 / 2}$ (full width at half maximum) with widths of approximately $17{ }^{\circ} \mathrm{C}$ (Table 4).

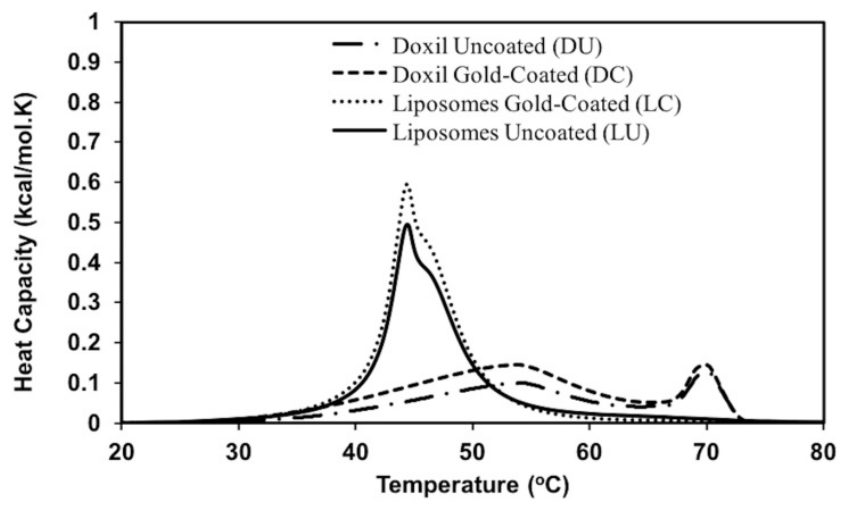

Figure 6. Isobaric thermograms of various formulations of doxorubicin after baseline subtraction. Doxil (DU), gold-coated Doxil (DC), Liposomes uncoated (LU) and Liposomes gold-coated (LC). Heating scans obtained at the rate of $1 \circ \mathrm{C} / \mathrm{min}$.

The DSC curves for LU and LC also exhibited a phase transition attributed to the main lipid phase transition (Figure 6). The transition for $\mathrm{LU}$ was observed at $44.5{ }^{\circ} \mathrm{C}$ with a shoulder at $46.2{ }^{\circ} \mathrm{C}$ while that of LC was seen at $45.1^{\circ} \mathrm{C}$ with a similar shoulder at $46.5^{\circ} \mathrm{C}$. The presence of gold had an impact on the first transition peak as indicated by a $0.6^{\circ} \mathrm{C}$ increase in the LC transition temperature in comparison to LU. 
Table 4. Thermodynamic parameters obtained from DSC measurements for lipid transitions the main lipid phase transition, determined by the greatest heat capacity (Figure 6).

\begin{tabular}{|c|c|c|c|c|c|}
\hline & $T_{m^{a}}\left({ }^{\circ} \mathrm{C}\right)$ & $\Delta T_{1 / 2} \mathrm{~b}\left({ }^{\circ} \mathrm{C}\right)$ & $\Delta H_{c a l}{ }^{c}(\mathrm{kcal} / \mathrm{mol})$ & $\Delta H_{V H^{\mathrm{d}}}(\mathrm{kcal} / \mathrm{mol})$ & $C U^{\mathrm{e}}$ \\
\hline DU & 51.6 & 16.8 & 1.93 & 43 & 22 \\
\hline DC & 52.3 & 16.5 & 2.45 & 42 & 17 \\
\hline LU & 44.5 & 8.05 & 3.76 & 504 & 134 \\
\hline LC & 45.1 & 6.70 & 4.50 & 567 & 126 \\
\hline $\begin{array}{l}\text { a } T_{m:} \\
\text { b } \Delta T \\
\text { c } \Delta H \\
\text { d } \Delta H \\
\text { е } C U\end{array}$ & $\begin{array}{l}\text { transition } \\
/ 2: \text { width } \\
\text { cal: calorin } \\
V_{H}: \text { van't I } \\
\text { cooperat }\end{array}$ & $\begin{array}{l}\text { emperature } \\
\text { the endoth } \\
\text { tric enthalp } \\
\text { ff enthalpy } \\
\text { e unit }\end{array}$ & $m$ at half the heigh & & \\
\hline
\end{tabular}

The peak attributed to the presence of doxorubicin crystals in the DU and DC samples was absent in the LU and LC samples. This result can be attributed to the thermo-sensitivity of liposomal formulation which has a phase transition temperature of $44.5^{\circ} \mathrm{C}$ and $45.1^{\circ} \mathrm{C}$ for LU and LC respectively. At the phase transition drug leakage is expected to occur, a fact that is shown in Figure 4 where leakage occurs at $42{ }^{\circ} \mathrm{C}$. Since the doxorubicin crystals peak occur at $70{ }^{\circ} \mathrm{C}$, after leakage at $42^{\circ} \mathrm{C}$, it is unlikely that there is drug present to generate a transition peak in the LU and LC samples. The endotherm widths of LU and LC were narrower than those seen for DU and DC samples with values of $\Delta T_{1 / 2}=8.05{ }^{\circ} \mathrm{C}$ and $6.70{ }^{\circ} \mathrm{C}$ for LU and LC respectively.
The calorimetric enthalpy for LC was slightly greater than that of LU and this difference is reflected in the calculated values of the van't Hoff enthalpies, 504 and $567 \mathrm{kcal} / \mathrm{mol}$ for LU and LC respectively. Despite this difference, both the uncoated and the gold-coated liposome samples had similar cooperative unit values of 134 units (LU) and 126 units (LC) (Table 4).

Overall, the Doxil samples exhibited lower calorimetric and van't Hoff enthalpies than the liposomes samples. Specifically, the van't Hoff enthalpies for the DU and DC samples were 12-fold lower than that reported for LU. Consequently, the Doxil cooperative unit sizes were also smaller at 22 and 17 units for DU and DC respectively.

\section{Cell Viability}

Melanoma cells were treated with liposome formulations and free doxorubicin. Cell viability was then determined using the resazurin assay. Three different conditions were tested. First, cells were treated with liposomes or free doxorubicin but were not exposed to hyperthermia or laser illumination (Figure 7A). The second condition utilized liposomal formulations or free doxorubicin at hyperthermia at $42{ }^{\circ} \mathrm{C}$ for 20 minutes (Figure 7B). Third, cells were
A

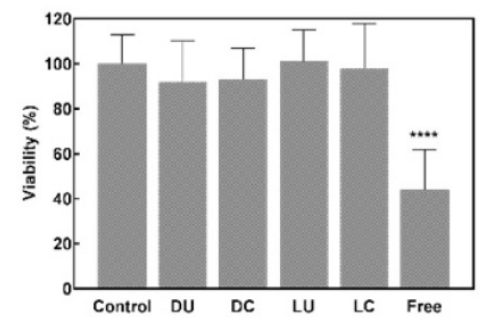

B

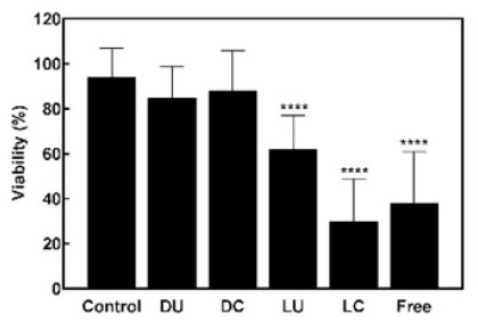

C

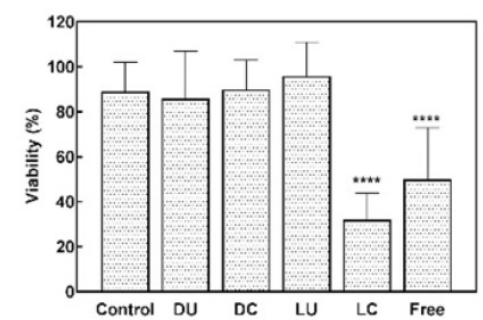

D

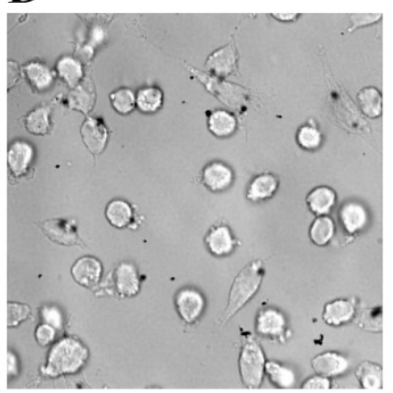

A375 Cells

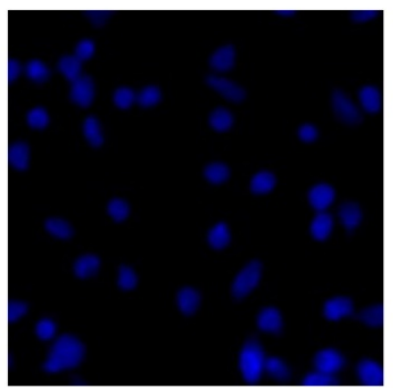

Cell Nuclei

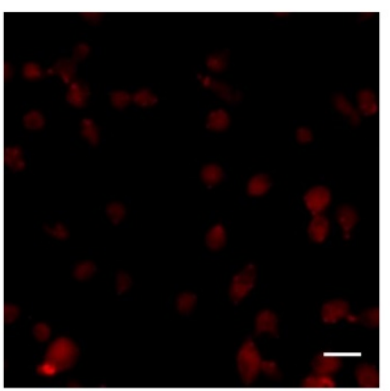

Doxorubicin Loaded Gold-coated Liposomes

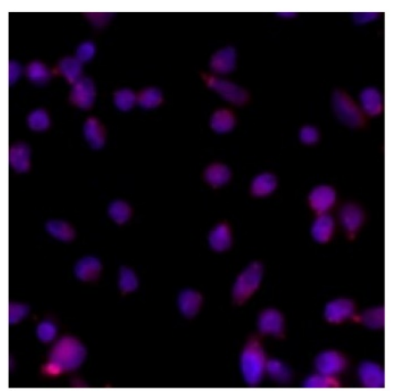

Merged

Figure 7. Cell viability on exposure of A375 cells to doxorubicin loaded liposomes and Doxil. A. Cells were treated with liposomes that were not exposed to stimulus (no stimulus). B. Cells treated with liposomes exposed to hyperthermia $\left(42{ }^{\circ} \mathrm{C}\right)$ for 20 minutes. C. Cells treated with liposomes exposed to $760 \mathrm{~nm}$ laser illumination for 2 minutes. Cells were then treated with resazurin to determine cell viability. All results were normalized to control cells that were not exposed to any drug. Error bars indicate standard deviation $(n=8)$, **** $p<0.0001$ vs. control (One way ANOVA followed by Dunnet's multiple comparisons test).D. Fluorescence images of uptake of loaded gold-coated (LC) liposome uptake in melanoma cells. Blue represents the nuclei and red represents loaded gold-coated (LC) liposomes. Scale bar represents $25 \mu \mathrm{m}$. 
exposed to liposome formulations or free drug, and were irradiated with laser at $760 \mathrm{~nm}$ for two minutes (Figure 7C). In all instances the free drug reduced cell viability to under $50 \%$. Moreover, in all cases, Doxil sample, both uncoated (DU) and gold-coated (DC), did not have significant effect on cell viability as indicated by greater than $85 \%$ viability in all samples post treatment.

The cells exposed to uncoated, doxorubicinloaded liposomes (LU) behaved as expected; upon exposure of formulation to hyperthermia cell viability reduced to $60 \%$. On the other hand, with laser exposure as well as in the absence of any stimulus, cell viability remained unaffected by LU. Doxorubicin appears to remain stably encapsulated in the gold-coated liposomes (LC) in the absence of hyperthermia and laser illumination as indicated by the lack of effect on viability (Figure 7A). However, upon application of either stimulus (hyperthermia or $760 \mathrm{~nm}$ laser illumination), decreasing cell viability to under $40 \%$ indicates controlled release of doxorubicin from LC (Figure 7B and 7C).

\section{Discussion}

Plasmon resonant gold nanoparticles find applications in photothermal therapies and cancer diagnostics [30,31]. In this work, we combined two phenomena, photothermal conversion in plasmon resonant gold coating and thermal sensitivity of certain liposome compositions, to achieve activation of the drug release process. The gold-coated liposomes are capable of a two-step process whereby illumination with NIR light activates content release by the energy conversion from light to heat, which subsequently produces the liposome phase transition from the gel to liquid crystalline state. This transition effectively increases the bilayer permeability with ensuing release of content from the liposomal core [32]. Here, we demonstrated that plasmon resonant liposomes enable temporal control of doxorubicin release that is not possible in Doxil, the current FDA-approved liposomal formulation of doxorubicin.

All the formulations investigated are stable at $\mathrm{pH} 7.4$ and $37{ }^{\circ} \mathrm{C}$ (Figure 3). Doxil samples, both uncoated (DU) and gold coated (DC), showed no evidence of leakage on exposure to mild hyperthermia or laser illumination (Figures 4 and 5). These data fall in line with well-known stability of Doxil, the feature that allows the accumulation of the liposomal drug at the tumor site via the enhanced permeability and retention (EPR) effect [33, 34]. Delivery systems such as these rely on the local environment for content release via a passive diffusive process, difference in $\mathrm{pH}$, or the presence of enzymes or other molecules that assist in the mechanical breakdown of the lipid membrane. They generally lack a mechanism for precise spatial and temporal activation of release. In contrast, thermosensitive liposomes, both uncoated (LU) and gold-coated (LC), while also stable at physiological conditions, release their content upon mild hyperthermia. However, upon exposure to laser illumination gold-coated liposomes (LC) release doxorubicin at a remarkably high rate, three orders of magnitude greater than that observed with no illumination.

Quantitative analysis of release was performed by determination of permeability coefficient and its changes in response to mild hyperthermia or laser illumination. The permeability coefficients remained approximately the same in all samples at $37^{\circ} \mathrm{C}$ as well as in heated $\left(42{ }^{\circ} \mathrm{C}\right)$ and irradiated $(760 \mathrm{~nm})$ Doxil. However, permeability coefficient of LU and LC was strongly dependent on stimulus applied. Both compositions were responsive to hyperthermia, with permeability coefficient increasing by a factor of 100 when the temperature was raised from $37^{\circ} \mathrm{C}$ to $42^{\circ} \mathrm{C}$. Upon laser illumination at $37{ }^{\circ} \mathrm{C}$, the uncoated formulation was far less responsive. A dramatic change was observed for the gold-coated formulation; the permeability coefficient increased three orders of magnitude and reached $0.60 \times 10^{-7} \mathrm{~cm} / \mathrm{s}$, just an order of magnitude below the permeability coefficient of urea across a red blood cell membrane, $5.7 \times 10^{-7} \mathrm{~cm} / \mathrm{s}$ [35]. For comparison, the permeability coefficient we achieved is three orders of magnitude greater than the permeability coefficient of doxorubicin through a lipid membrane composed of DPPC and $4 \%$ DSPE-PEG, reported by others at $3.4 \times 10^{-10} \mathrm{~cm} / \mathrm{s}$ [36].This rapid release sets this system apart from other liposomal drug delivery systems that utilize hyperthermia [37], NIR-responsive dyes embedded within the lipid membrane [38], or the co-injection of gold nanoantennas and thermosensitive drug delivery system [39] to regulate drug release.

As determined by differential scanning calorimetry (DSC), the addition of gold to liposomes caused a $0.6{ }^{\circ} \mathrm{C}$ increase in the phase transition temperature. Wang et al. investigated the phase transition of citrate-capped gold nanoparticles adsorbed to the liposome surface. While employing a different method of gold addition to the lipid surface, they have also reported gold interaction with lipids resulting in an increase in phase transition temperature $[40,41]$. This effect is also reflected in a $1.4{ }^{\circ} \mathrm{C}$ narrowing in peak width, an indication of an increase in cooperativity. On the other hand, the thermograms obtained for Doxil display much lower cooperativity of its phase transition. These results are in general agreement with recently published data 
[42] indicating that Doxil does not undergo a well pronounced thermotropic phase transition. In accord with the prevailing model [43], the lack of phase transition inhibits temperature-induced reorganization of lipids at the main phase transition, diminishing content release from such compositions.

Near-infrared illumination of plasmon resonant thermosensitive liposomes act as a permeability switch, changing the membrane permeability coefficient over a broad range of values that is not available by simply increasing temperature of the media. This renders the plasmon resonant liposomes particularly suitable for applications requiring instantaneous release of contents. The remarkable change of permeability coefficient we observe in gold-coated thermosensitive liposomes may be explained by the high thermal conductivity of gold. The heat flow toward the lipid membrane, generally governed by the Fourier Law, is directly related to the thermal conductivities of materials in contact, therefore $k_{\text {lipid }}=0.4 \mathrm{~W} / \mathrm{m} \times \mathrm{K}$ and either $k_{\text {water }}=0.6$ $\mathrm{W} / \mathrm{m} \times \mathrm{K}$ or $k_{\text {gold }}=314 \mathrm{~W} / \mathrm{m} \times \mathrm{K}$, yielding a greater heat flow across the gold-lipid interface when compared to the water-lipid interface [44]. Therefore, the use of gold nanoparticles as a sensitizer for activation of a thermal process ensures a much quicker and complete change of permeability than that observed with hyperthermia of uncoated liposomes or using various types of molecular or organic sensitizer.

The impact of this photoactivated release of doxorubicin on viability of melanoma cells was evaluated using resazurin, a fluorescent indicator producing an irreversible color change due to metabolic activity. In the absence of release stimulus, doxorubicin remains stably encapsulated in all liposome formulations, including Doxil, and therefore does not impact cell viability (Figure 7A). Mild hypothermia $\left(42{ }^{\circ} \mathrm{C}\right)$ significantly reduced viability of cells exposed to uncoated liposomes (LU) and gold-coated liposomes (LC), both loaded with doxorubicin. However, light-activated cytotoxicity was observed only with LC. While LC elicits similar toxic effects upon laser illumination as hyperthermia, with both reducing cell viability by nearly $70 \%$, the time associated with this effect is significantly different. It requires 2 minutes of near-infrared illumination or 20 minutes of hypothermia to produce a similar reduction of cell viability.

In comparison to LU, LC show higher permeability (Fig 4) and produce higher cytotoxicity (Fig 7B) upon exposure to mild hyperthermia. This difference can be attributed to changes in the lipid membrane behavior caused by the attached gold clusters. Note that DSC indicates a rather mild difference between LU and LC phase transition enthalpy (Fig 6). The data suggest that gold-coated liposomes as well as uncoated liposomes undergo a similar phase transition upon hyperthermia, both leading to increased permeability. However, it appears that the ensuing rearrangement of lipids modified with gold clusters (LC) disturbs the membrane organization more, and produces permeability greater than that of made of unmodified lipid (LU), leading to faster release of contents from LC.

The unique properties of plasmon resonant liposomes are not limited to applications in drug delivery but can in fact translate to include multifunctional, theranostic applications. Studies have shown that gold nanoparticles, within the size range described in our model, can be used as contrast agents for imaging modalities including X-ray [45] and computed tomography (CT) [46] used in the detection of different mammalian tumors. Importantly for biomedical applications, gold coated liposomes we introduced are biodegradable to sizes that are compatible with renal clearance [19,47]. Our own work demonstrates the possibility of using gold coated liposomes to study cellular communication in vitro [25] or for imaging vasculature of the brain in vivo [48]. Together, these observations point to the possibility of developing novel multifunctional, theranostic applications based on gold-coated liposomes.

\section{Conclusions}

Controlled release of doxorubicin, and possibly other anthracyclines, can be accomplished by photothermal conversion in gold coated liposomes. The release initiated by near-infrared laser produces a faster onset of cytotoxicity than that achieved by hyperthermia. Successful implementation of this technique requires a specialized thermally-responsive lipid composition, whereas the commercial formulation of doxorubicin is not directly amenable to this mechanism of controlled release.

\section{Abbreviations}

DPPC: 2-dipalmitoyl-sn-glycero-3-phosphocholine; HSPC: Hydrogenated Soy L-a-phosphatidylcholine; DPPE-PEG: 1,2-dipalmitoyl-sn-glycero-3phosphoethanlamine-N-[methoxy(polyethyleneglycol ); CHOL: Cholesterol; DSPE-PEG: 1,2-distearoyl-snglycero-3-phosphoethanlamine-N-[methoxy(polyethy leneglycol); HBS: HEPES Buffered Saline; EE: Encapsulation Efficiency; LU: Loaded Uncoated; LC: Loaded Gold-coated; DU: Unloaded Doxil; DC: Gold-coated Doxil; DSC: Differential Scanning Calorimetry; EPR: Enhanced Permeability and Retention; NIR: Near Infrared. 


\section{Acknowledgements}

This work was supported by the National Institutes of Health [P01CA027502].

\section{Competing Interests}

The authors have declared that no competing interest exists.

\section{References}

1. Siegel RL, Miller KD, Jemal A. Cancer Statistics, 2017. CA Cancer J Clin. 2017; 67: 7-30.

2. von Eschenbach AC. NCI sets goal of eliminating suffering and death due to cancer by 2015. J Natl Med Assoc. 2003; 95: 637-9.

3. Morgan GW, Ward R, Barton M. The contribution of cytotoxic chemotherapy to 5-year survival in adult malignancies. Clin Oncol. 2004; 16: 549-60.

4. Chabner BA, Jr TGR. Timeline: Chemotherapy and the war on cancer. Nat Rev. 2005; 5: 65-72.

5. Hon YY, Evans WE. Making TDM work to optimize cancer chemotherapy: a multidisciplinary team approach. Clin Chem. 1998; 44: 388-400.

6. Gabizon A, Shmeeda H, Grenader T. Pharmacological basis of pegylated liposomal doxorubicin: impact on cancer therapy. Eur J Pharm Sci. 2012; 45: 388-98.

7. Barenholz Y. Doxil - the first FDA-approved nano-drug: lessons learned. J Control. Release. 2012; 160: 117-34.

8. Gabizon A, Shmeeda H, Barenholz Y. Pharmacokinetics of pegylated liposomal doxorubicin: Review of animal and human studies. Clin Pharmacokinet. 2003; 42: 419-36.

9. O'Brien ME, Wigler N, Inbar M, Rosso R, Grischke E, Santoro A, et al. Reduced cardiotoxicity and comparable efficacy in a phase III trial of pegylated liposomal doxorubicin $\mathrm{HCl}$ (CAELYX/Doxil) versus conventional doxorubicin for first-line treatment of metastatic breast cancer. Ann Oncol. 2004; 15: 440-9.

10. Kobayashi H, Turkbey B, Watanabe R, Choyke PL. Cancer drug delivery: considerations in the rational design of nanosized bioconjugates. Bioconjug. Chem. 2014; 25: 2093-100.

11. Minko T, Dharap SS, Pakunlu RI, Wang Y. Molecular targeting of drug delivery systems to cancer. Curr Drug Targets. 2004; 5: 389-406.

12. Ruoslahti E, Bhatia SN, Sailor MJ. Targeting of drugs and nanoparticles to tumors. J Cell Biol. 2010; 188: 759-68.

13. Qin Y, Chen J, Bi Y, Xu X, Zhou H, Gao J, et al. Near-infrared light remote-controlled intracellular anti-cancer drug delivery using thermo/ $\mathrm{pH}$ sensitive nanovehicle. Acta Biomater. 2015; 17: 201-9.

14. Landon CD, Park JY, Needham D, Dewhirst MW. Nanoscale drug delivery and hyperthermia: The materials design and preclinical and clinical testing of low temperature-sensitive liposomes used in combination with mild hyperthermia in the treatment of local cancer. Open Nanomed J. 2011; 3: 38-64.

15. Mura S, Nicolas J, Couvreur P. Stimuli-responsive nanocarriers for drug delivery. Nat Mater. 2013; 12: 991-1003.

16. Luo D, Carter KA, Razi A, Geng J, Shao S, Giraldo D, et al. Doxorubicin encapsulated in stealth liposomes conferred with light-triggered drug release. Biomaterials. 2016; 75: 193-202.

17. Troutman TS, Leung SJ, Romanowski M. Light-induced content release from plasmon-resonant liposomes. Adv Mater. 2009; 21: 2334-8.

18. Leung SJ, Romanowski M. NIR-activated content release from plasmon resonant liposomes for probing single-cell responses. ACS Nano. 2012; 6: 9383-91.

19. Troutman TS, Barton JK, Romanowski M. Biodegradable plasmon resonant nanoshells. Adv Mater. 2008; 20: 2604-8.

20. Leung SJ, Kachur XM, Bobnick MC, Romanowski M. Wavelength-selective light-induced release from plasmon resonant liposomes. Adv Funct Mater. 2011; 21: 1113-21.

21. Leung SJ, Romanowski M. Biomedical Applications: Composite nanocapsules, dielectric Properties. Dekker Encyclopedia of Nanoscience and Nanotechnology. 3rd ed. Lyshevski SE, editor. Taylor \& Francis; 2014; 336-346

22. Fomina N, Sankaranarayanan J, Almutairi A. Photochemical mechanisms of light-triggered release from nanocarriers. Adv Drug Deliv Rev. 2012; 64: 1005-20.

23. Kwon HJ, Byeon Y, Jeon HN, Cho SH, Han HD, Shin BC. Gold cluster-labeled thermosensitive liposmes enhance triggered drug release in the tumor microenvironment by a photothermal effect. J Control Release. 2015; 216: 132-9.

24. Leung SJ, Romanowski M. Molecular catch and release: controlled delivery using optical trapping with light-responsive liposomes. Adv Mater. 2012; 24: 6380-3.

25. Orsinger G V., Williams JD, Romanowski M. Focal activation of cells by plasmon resonance assisted optical injection of signaling molecules. ACS Nano. 2014; 8: 6151-62.

26. Timko BP, Kohane DS. Drug-delivery systems for tunable and localized drug release. Isr J Chem. 2013; 53: 728-36.
27. de Smet M, Langereis S, den Bosch S Van, Grüll H. Temperature-sensitive liposomes for doxorubicin delivery under MRI guidance. J Control Release. 2010; 143: 120-7.

28. Clary L, Verderone G, Santaella C, Vierling P. Membrane permeability and stability of liposomes made from highly fluorinated double-chain phosphocholines derived from diaminopropanol, serine or ethanolamine. Biochim Biophys Acta. 1997; 1328: 55-64.

29. McElhaney RN. The use of differential scanning calorimetry and differential thermal analysis in studies of model and biological membranes. Chem Phys Lipids. 1982; 30: 229-59.

30. Pissuwan D, Valenzuela SM, Cortie MB. Therapeutic possibilities of plasmonically heated gold nanoparticles. Trends Biotechnol. 2006; 24: 62-7.

31. Huang X, El-Sayed IH, Qian W, El-Sayed MA. Cancer cell imaging and photothermal therapy in the near-infrared region by using gold nanorods. J Am Chem Soc. 2006; 128: 2115-20.

32. Papahadjopoulos SD, Jacobson K, Nir S, Isac T. Phase transitions in phospholipid vesicles fluorescence polarization and permeability measurements concerning the effect of temperature and cholesterol. Biochim Biophys Acta. 1973; 311: 330-48.

33. Yuan F, Leunig M, Kun Huang S, Berk DA, Papahadjopoulos D, Jam RK. Microvascular permeability and interstitial penetration of sterically stabilized (stealth) liposomes in a human tumor xenograft' reveal heterogeneous distribution of liposomes in tumor interstitium. Cancer Res. 1994; 54: 3352-6.

34. Huang SK, Lee KD, Hong K, Friend DS, Papahadjopoulos D. Microscopic localization of sterically stabilized liposomes in colon carcinoma-bearing mice. Cancer Res. 1992; 52: 5135-43.

35. Wood RE, Jr FPW, Morgan HE. Glucose permeability of lipid bilayer membranes. Biochim Biophys Acta. 1968; 163: 171-8.

36. Mills JK, Needham D. Lysolipid incorporation in dipalmitoylphosphatidylcholine bilayer membranes enhances the ion permeability and drug release rates at the membrane phase transition. Biochim Biophys Acta. 2005; 1716: 77-96.

37. Needham D, Anyarambhatla G, Kong G, Dewhirst MW. A new temperature-sensitive liposome for use with mild hyperthermia: characterization and testing in a human tumor xenograft model. Cancer Res. 2000; 60: 1197-201

38. Yuan A, Huan W, Liu X, Zhang Z, Zhang Y, Wu J, et al. NIR light-activated drug release for synergetic chemo-photothermal therapy. Mol Pharm. 2017; 14: $242-51$.

39. Ou Y-C, Webb JA, Faley S, Shae D, Talbert EM, Lin S, et al. Gold nanoantenna-mediated photothermal drug delivery from thermosensitive liposomes in breast cancer. ACS Omega. 2016; 1: 234-43.

40. Wang F, Curry DE, Liu J. Driving adsorbed gold nanoparticle assembly by merging lipid gel/ fluid interfaces. Langmuir. 2015; 31: 13271-4.

41. Wang F, Liu J. Self-healable and reversible liposome leakage by citrate-capped gold nanoparticles: probing the initial adsorption/desorption induced lipid phase transition. Nanoscale. 2015; 38: 15599-604.

42. Wei X, Cohen R, Barenholz Y. Insights into composition/structure/function relationships of Doxil gained from "high-sensitivity" differential scanning calorimetry. Eur J Pharm Biopharm. 2016; 104: 260-70.

43. Gaber MH, Hong K, Huang SK, Papahadjopoulos D. Thermosensitive sterically stabilized liposomes: formulation and in vitro studies on mechanism of doxorubicin release by bovine serum and human plasma. Pharm Res. 1995; 12: $1407-16$

44. Urban P, Kirchner SR, Mühlbauer C, Lohmüller T, Feldmann J. Reversible control of current across lipid membranes by local heating. Sci Rep. 2016; 6: 22686.

45. Hainfeld JF, Slatkin DN, Focella TM, Smilowitz HM. Gold nanoparticles: a new X-ray contrast agent. Br J Radiol. 2006; 79: 248-53.

46. Kattumuri V, Katti K V., Boote E, Kannan R, Casteel S, Churchill R. Stabilized gold nanoparticle and contrast agent. US Patent US 9,549,998; January 24, 2017.

47. Choi HS, Liu W, Misra P, Tanaka E, Zimmer JP, Itty Ipe B, et al. Renal clearance of nanoparticles. Nat Biotechnol. 2007; 25: 1165-70.

48. Watson JR, Garland S, Romanowski M. Intraoperative visualization of plasmon resonant liposomes using augmented microscopy. In: Pogue BW, Gioux S, ed. Proc SPIE 10049 Molecular-Guided Surgery. Molecules, Devices and Applications III. International Society for Optics and Photonics; 2017: 100490L. 